EMTs, and the operational aspects and support behind the scenes to deploy a team.

Methods: Analysis of operational activities and support for predeployment, deployment, and post-deployment phases including preparedness through innovation, collaboration, development, and maintenance of a high-performance team and cache. Results: The analysis of operational activities behind the scenes of deploying EMT maps the unique complexities of maintaining and deploying a high-performance team at all stages of deployment, demonstrating the success of a team in the field is attributed to the support and activities of the team back home to deploy them.

Discussion: There is substantial preparation and behind the scenes operational activities that are undertaken to deploy and support a deployed EMT. Lessons learned from each deployment build on the operational capacity of staff deploying a team and on the future directions, innovations, and practices of a deployed team in the field.

Prehosp Disaster Med 2019;34(Suppl. 1):s159-s160

doi:10.1017/S1049023X19003613

\section{Preparing Health Care Professionals for Public Health Disaster Management} Regina Rigatto Witt $P h D^{1}$, Alexandre Barbosa Oliveira $P h D^{2}$, Elaine Silva Miranda $P h D^{3}$, Cristianne Maria Famer Rocha $P h D^{1}$, Collective health student Natalia Silva Pires ${ }^{1}$, Nursing student Laura Lucas Silva ${ }^{1}$, Ms Marcio Haubert Silva ${ }^{1}$, Ms Potiguara Oliveira Paz ${ }^{1}$, Ms Carla Daiane Silva Rodrigues ${ }^{1}$, Ms Robriane Prosdocimi Menegat ${ }^{1}$, Ms Walnice Jung ${ }^{1}$

1. Universidade Federal do Rio Grande do Sul, Porto Alegre, Brazil

2. Universidade Federal do Rio de Janeiro, Rio de Janeiro, Brazil

3. Universidade Federal Fluminense, Rio de Janeiro, Brazil

Introduction: Disasters are a major challenge for public health because of damage caused by death, injury, or illness that exceeds health services' ability to respond. Health professionals and students require awareness and understanding of particular aspects of disaster planning, mitigation, response, or recovery. In Brazil, despite the increase in the number and intensity of disasters, there is no formal acceptance regarding the need to integrate disaster content into curriculum guidelines (1)

Aim: To develop and test referential and models for disaster management health professional education.

Methods: Competence-based education has been proposed. The methodology adopted was developed by the Association (2) and adapted to be used in the Brazilian context. An initial literature search was performed in MEDLINE via PubMed, Google Scholar, Lilacs, and Scielo databases using disaster and competencies as descriptors.

Results: Articles and documents in Portuguese, Spanish, and English were identified for: public health (21), nursing (20), multi-professional (16), psychology (4), pharmacy (4), dentistry (2), medicine (1), veterinary (2), and nutrition (1). Data were organized according to a proposal from the literature (3) Selection of benchmarks for the preparation of education models identified 27 referential, three of them developed in Brazil. Discussion: Application and evaluation of the methodology developed with undergraduate students of the Federal
University of Rio Grande do Sul consisted of an initiative to prepare health care professionals for disaster management.

\section{References:}

1. Witt RR, Gebbie KM. Tailoring curricula to fit health professionals needs in a disaster: a proposal for Brazilian nurses. Rev Gaúcha Enferm. 2016;37(1).

2. Association for Prevention Teaching and Research (US); Columbia University, School of Nursing, Center for Health Policy. Competency-to-curriculum toolkit. Washington: APTR; 2008.

3. Schor, KW. and Altman, BA. Proposals for aligning disaster health competency models. Disaster Medicine and Public Health Preparedness. 2013;7(1):8-12.

Prehosp Disaster Med 2019;34(Suppl. 1):s160

doi:10.1017/S1049023X19003625

\section{The Problems on Your Desk: A Research Study to Define and Describe Paramedic Practice in Canada Dr. Ron Bowles}

Justice Institute Of British Columbia, New Westminster, Canada

Introduction: Paramedicine is a rapidly evolving profession, growing from its initial role of providing emergency care and transportation of the sick and injured into a broad discipline providing a wide range of care in multiple practice settings, yet the field is relatively unexplored. Much of the research in the field centers on patient care, often from the perspective of emergency medicine. A growing body of literature describing the discipline itself is largely descriptive.

Aim: This interactive presentation describes and contributes to an applied research project that will define and describe Canadian paramedic practice. The research will develop frameworks, common taxonomy and designs, and evidence to support development of a national Canadian Paramedic Information System.

Methods: This two-year mixed methods study is gathering data from a literature review, stakeholder workshops, and key informant interviews. The project will develop "user cases" that explore the issues and challenges facing Canadian paramedic stakeholders and identify the information and data required to address those issues.

Results: The presentation will present initial findings that describe core concepts, data/knowledge structures, and models that are foundational to understanding and informing current and emerging paramedic practice. It will explore this data in relation to operational needs of practitioners, operations, communities, and stakeholders to inform decision-making, guiding policy and direction, and advancing the profession. Lastly, it will develop explanatory principles, models, and relationships in a conceptual framework that describes paramedic practice.

Discussion: The study will develop models and core data sets that guide research and support policy development at local and national levels, and inform operational and strategic decisionmaking. The presentation will provide an overview of the research and findings to date. Participants will engage in activities that explore the user-cases and selected findings, applying 
the results of the study through the lens of their own backgrounds and operational contexts.

Prehosp Disaster Med 2019;34(Suppl. 1):s160-s161

doi:10.1017/S1049023X19003637

\section{Putting the Theoretical to Practical Use Dr. Eli Jaffe \\ Magen David Adom, Tel Aviv, Israel}

Introduction: Managing an MCI (Mass Casualty Incident) can be a daunting task for emergency responders. Effective management can be a matter of life and death but can be directly impacted by the feelings of the incident commander.

Aim: Students were trained to be incident commanders, then following the course were given a survey. In the days following the training, an MCI occurred involving a train full of passengers. The students were then given another survey to assess their readiness following the practical use of their studies.

Methods: Students were given a survey to determine their mean level of confidence in managing MCIs prior to training, and following the training. Following the training, there was an increase in confidence. After the training, there was an MCI in which their theoretical knowledge was put to the test.

Results: The pre-training self-efficacy mean scores of younger students ( $M=3.5, S D+0.23)$ increased after the training $(\mathrm{M}=3.8, \mathrm{SD}+0.28)$ and rose even more following the presentation of the Turin train accident $(\mathrm{M}=4, \mathrm{SD}+0.26)$. While a similar increase in self-efficacy was found among the more mature students post-training compared to the level prior to the training $(\mathrm{M}=3.7, \mathrm{SD}+0.44$ versus $\mathrm{M}=3.4, \mathrm{SD}+0.56)$, the mean selfefficacy score of the mature students decreased following the presentation of the Turin train accident to the pre-training level $(\mathrm{M}=3.4, \mathrm{SD}+0.51)$.

Discussion: Mean scores of self-efficacy and confidence in managing MCIs were found to be higher among medical students that were previously trained in coping with MCIs compared to medical students who participated in such a training program for the first time.

Prehosp Disaster Med 2019;34(Suppl. 1):s161

doi:10.1017/S1049023X19003649

A Qualitative Study of Household Emergency Preparedness of the Elderly and the Medically Frail Living in Coastal Urban Environments

Dr. Tara Heagele

The City University of New York, New York, United States

Introduction: As more chronically ill people are living in the community and disasters are occurring frequently, the elderly and the medically frail vulnerable populations are experiencing significantly more disaster-related morbidity and mortality than the rest of the population. A failure to adequately address these vulnerabilities has been shown to have negative effects on the response to the disaster and the community as a whole.

Aim: The purpose of this research was to understand how older and/or medically frail adults have experienced disaster and how this experience impacts what they do now to prepare for disaster. A second purpose was the generation of theory regarding the process through which community members prepare for disasters.

Methods: This study employed a qualitative descriptive methodology, Situational Analysis, to explore the social processes of disaster preparedness in older and/or medically frail adults.

Results: The core category was "Experience is the Best Teacher." Based on the findings, it was theorized that these community members are generally considered unprepared for disaster. Their lack of preparedness is due in large part to a lack of education on how best to prepare. Once educated, motivation for self-responsibility of household emergency preparedness can be expected. Community interventions like distributing disaster supply kits and offering evacuation assistance help overcome their situational impediments to preparedness and provide the best chance for these community members to survive disasters without becoming ill or injured or experiencing a decline in their baseline functional status. If these community members are incidentally prepared, it is largely due to their past experience with disaster or their professional experience.

Discussion: The results from this study could inform emergency plans and policy efforts to better meet the needs of elderly and medically frail community members during disaster.

Prehosp Disaster Med 2019;34(Suppl. 1):s161

doi:10.1017/S1049023X19003650

\section{A Quantitative Study of the Dimensional Change of Inferior Vena Cava on Computed Tomography During Acute Hemorrhage Shock}

Dr. Yang $L i$

Army Medical Center of PLA, Chongqing, China

Introduction: The collapsed inferior vena cava (IVC) in computed tomography (CT) images can be found in patients with hypovolemic shock, making it an attractive diagnostic sign in early treatment of trauma patients. However, current research results are controversial.

Aim: To examine the dimensional change of IVC during acute hemorrhage through a volume controlled acute hemorrhagic shock model in swine.

Methods: Volume controlled hemorrhage was performed in 10 adult Bama minipigs. Enhanced CT scan and hemodynamic monitoring were performed when the cumulative blood loss volume reached $0 \%, 10 \%, 20 \%, 30 \%$, and $40 \%$. The transverse diameter (T) and anteroposterior diameter (AP) of IVC were measured in axial images. Hemodynamic parameters were obtained with a Pulse Contour Cardiac Output (PiCCO) hemodynamic monitor device. Arterial blood samples were also collected for artery blood gas analysis at each time point.

Results: As the blood loss volume increased, the collapsibility (T/AP) and cross section area (CSA) of IVC significantly changed in hepatic level and pre-renal level. The significant decrease of the CSA of IVC (shrink) occurred early when the blood loss volume reached only $10 \%$.

Discussion: The IVC shrank early but collapsed late during acute hemorrhages in swine. The collapsed IVC on CT scans 